\title{
New genetic sources for orange color in cucumber (Cucumis sativus L.) fruit flesh
}

Brian M. Waters ${ }^{1}$, HaeJin Kim $^{2,3}$, and Keenan Amundsen ${ }^{1}$

${ }^{1}$ Department of Agronomy and Horticulture, University of Nebraska-Lincoln, Lincoln, NE USA $68583,{ }^{2}$ Department of Biochemistry, University of Nebraska-Lincoln, Lincoln, NE USA 68588, ${ }^{3}$ Current address: Pairwise Plants, Research Triangle Park, NC 27709

\section{Abstract}

Most cucumber varieties have fruits with white flesh, which is devoid of ß-carotene and has a low concentration of total carotenoids. Carotenoids are important nutrients for humans and animals. Thus, developing cucumber varieties with orange flesh could provide a new nutritionally enhanced food source. Some cucumbers with yellow and orange flesh have been described, but there are others that have not been studied. Here, we used three cucumber PI lines, reported to produce colored fruits, from the USDA National Plant Germplasm System to generate three $F_{2}$ populations. Fruits from the $F_{2}$ populations with colored flesh (green, yellow, or orange) were pooled, and an equal number of fruits with white flesh were pooled. RNA was isolated from the pools and used for RNA sequencing to determine gene expression differences and to identify SNPs in each pool. The orange color of the cucumber fruits was confirmed to be due to ß-carotene. There were no clear expression patterns for genes of the carotenoid biosynthesis pathway that would suggest that their expression controlled the coloration of fruits. Mutations in carotenoid biosynthesis genes also did not explain the variation. However, we detected a SNP in the homolog of the Or gene that is responsible for ß-carotene accumulation in orange cauliflower. This genetic basis is different from that of previously studied orange cucumbers, but our results suggest that $O r$ is not the only factor. These results provide the basis for future studies for breeding orange cucumbers for commercial or home garden production.

\section{Introduction}

Cucumber (Cucumis sativus L.) is a popular and widely consumed fruit. Most cucumber varieties have green skin (exocarp) and white flesh (mesocarp and endocarp) (Che and Zhang, 2019). A number of genes that control exocarp color, which can also be orange, yellow, or cream-colored, have been described (Che and Zhang, 2019). Some cucumber varieties have colored flesh, such as yellow (Kooistra, 1971, Lu, H. W. et al., 2015) or orange (Simon and Navazio, 1997), due to carotenoid accumulation (Navazio and Simon, 2001, Cuevas et al., 2010), while the white flesh has no detectable ß-carotene and only trace amounts of total carotenoids (Kandlakunta et al., 2008). Carotenoids are yellow, orange, or red compounds with various functions in plants (Cazzonelli and Pogson, 2010). These carotenoid compounds also have 
important nutritional value for humans and animals, such as ß-carotene serving as a provitamin A compound (Nisar et al., 2015). Increasing the quantity of ß-carotene in cucumber could positively contribute to improved human nutrition and may provide a specialized horticultural product that is a colorful option for consumers. Colored cauliflower, carrots, and potatoes are popular with consumers. Orange cauliflower is caused by a mutation in a gene called Orange (Or) that encodes a DnaJ protein (Lu, S. et al., 2006). The or mutation results in development of chromoplasts (Lu et al., 2006) where ß-carotene accumulates (Li, L. and Yuan, 2013). SNPs in the homolog of this gene in melon, CmOr, can determine whether fruits are orange or green (Tzuri et al., 2015).

Another way that carotenoid levels can be controlled is through the carotenoid biosynthesis pathway. Expression levels of a plant phytoene synthase (PSY) and a phytoene desaturase ( $P D S)$, the first enzymes in the carotenoid synthesis pathway, are key contributors to ß-carotene levels in plants (Cazzonelli and Pogson, 2010). Additionally, decreased expression or mutation of proteins downstream of $ß$-carotene, such as $ß$-carotene hydroxylase (BCH), can also affect $ß$-carotene accumulation by disrupting its conversion to downstream compounds ( $Q$ i et al., 2013, Diretto et al., 2007).

Some cucumber germplasm that produces fruits with colored flesh have been studied. The line PI 200815 produces fruits with yellow flesh, but the genetic basis has not been determined (Kooistra, 1971, Lu et al., 2015). Orange colored germplasm has been identified and studied using the Xishuangbanna (XIS) cucumber from China (Simon and Navazio, 1997, Navazio and Simon, 2001, Cuevas et al., 2010, Bo et al., 2012). The orange coloration is due to ßcarotene (Cuevas et al., 2010), and a mutation in the $B C H 1$ gene was identified as the cause of orange coloration in a GWAS study (Qi et al., 2013).

Other than XIS and PI 200815, sources of colored flesh cucumber germplasm have not been studied, although there are some PI lines in the USDA National Plant Germplasm System that are described as producing fruits with colored flesh. The objectives of this study were to observe molecular differences in white and colored pools of cucumber fruits in $F_{2}$ populations derived from PI 200815, PI 163217, and PI 606066. Our approach was to perform RNA sequencing in these pools and observe differentially expressed genes (DEGs) and SNP differences between the pools, with an emphasis on genes of the carotenoid biosynthesis pathway and DnaJ chaperones. Our results indicate that the underlying basis for colored flesh differs from that of the XIS cucumber, and there may be multiple genes that will need to be identified to fully understand control of flesh color. These results are an important step for developing new sources of orange flesh genes for cucumber breeding.

\section{Materials and Methods}

\section{Plant materials}

Seeds of cucumber lines PI 606066, PI 163217, and PI 200815 were obtained through the USDA National Plant Germplasm System from the North Central Regional Plant Introduction Station in Ames, lowa. PI 606066 was collected from Madya Pradesh, India during the rainy season, and is described as having "orange flesh like C. melo" in the U.S. National Plant 
Germplasm System. PI 163217 was collected from Punjab, Pakistan, and is described as "flesh bright salmon". PI 200815 was collected from Mandalay, Myanmar, and is described as having "orange flesh".

Plants of each line were grown in a field and crossed with each other line. $F_{1}$ plants were grown in a greenhouse and self-pollinated to produce $F_{2}$ populations. On May 18, 2018, 25 plants of each population were transplanted into a field site on the University of Nebraska campus, and were allowed to be open-pollinated. Flowering began around the third week of June, and two representative fruits were harvested from each plant on July 23, 2018. Some plants had died, had been eaten by deer, or did not produce fruits. Fruit weight was recorded, as well as exocarp color and flesh color. Fruits without color (white flesh) and fruits with coloration (green, yellow, or orange) in the mesocarp and endocarp were selected for two pools of 17 fruits each. A cork borer was used to sample a plug of each fruit, which included exocarp, mesocarp, and endocarp, but not seeds or placental material. The plugs were frozen at $-80 \mathrm{C}$ until being ground together in a large mortar in liquid nitrogen. Four samples from each pool were used for RNA isolation using the Plant RNeasy kit (Qiagen).

\section{RNA-seq and SNP analysis}

The three best quality RNA samples were subjected to RNA sequencing using the $2 \times 75$ bp High-Output Kit on an Illumina NextSeq 500 (University of Nebraska Medical Center DNA Sequencing Core Facility, Omaha, NE). Raw sequencing reads were deposited to the short read archive National Center for Biotechnology Information database under the BioProject PRJNA546604 (https://www.ncbi.nlm.nih.gov/bioproject/?term=PRJNA546604). Trimmomatic 0.36 (Bolger et al., 2014) was used to remove low quality reads and reads contaminated with primers and adaptor sequences from the Illumina sequencing kit. During the trimming process, reads were also trimmed to a uniform $50 \mathrm{bp}$ length. The Gy14 v2 transcriptome was obtained from CuGenDB (Zheng et al., 2019). Forward sequencing reads from each pool were separately mapped to the Gy14 transcriptome using Bowtie 2.3 (Langmead and Salzberg, 2012). A read count data matrix was constructed and the Bioconductor edgeR package (Robinson et al., 2010) was used to infer differential gene expression between color and white pools. Differentially expressed genes were identified as described previously (Waters et al., 2014) from transcripts having at least 100 mapped reads and an edgeR false discovery rate $<0.1$.

Single nucleotide polymorphisms and relatively short insertions and deletions relative to the Gy14 transcriptome were identified using the default settings of Samtools 0.1 mpileup (Li, H. et al., 2009). Variants conserved among reps of one condition (color or white), but that differed from the other condition, were identified.

\section{Carotenoid analysis}

Cucumber flesh samples from one orange-fleshed, one white-fleshed, and one greenfleshed fruit were lyophilized. About $100 \mathrm{mg}$ of lyophilized sample was put into a screw-cap glass tube $(13 \times 100 \mathrm{~mm}, 9 \mathrm{~mL})$ and $2 \mathrm{ml}$ methanol: dimethylchloroether (9:1) was added. To extract carotenoids, the samples were ground with a homogenizer, then incubated at room 
temperature for $30 \mathrm{~min}$. The glass tubes were centrifuged, and $1 \mathrm{ml}$ solution was used for HPLC analysis. All samples were shielded from light during all processes. HPLC analysis was performed on an Agilent 12000 series system, fitted with a ProntoSIL 200-5 C30 column. The mobile phase was methanol: tert-butyl methyl ether $(80: 20, \mathrm{v} / \mathrm{v})$ at $1 \mathrm{~mL} / \mathrm{min}$ flow rate. Detection wavelength was $455 \mathrm{~nm}$.

\section{Results}

\section{Fruit size and color variation}

Fruit size (mass) varied in each $\mathrm{F}_{2}$ population (Fig. 1). The two populations that were generated with the PI 200815 parent produced smaller fruits than the third population. The PI 200815 X PI 163217 population fruits ranged from $511 \mathrm{~g}$ to $1740 \mathrm{~g}$, with an average mass of 998 g. The PI 606066 X PI 200815 population fruits ranged from $333 \mathrm{~g}$ to $1470 \mathrm{~g}$, with an average mass of $851 \mathrm{~g}$. The PI 606066 X PI 163217 population fruits ranged from $381 \mathrm{~g}$ to $2816 \mathrm{~g}$, with an average mass of $1495 \mathrm{~g}$. Fruit skin (exocarp) color also varied greatly (Fig. 2A, Table 1, Table S1). When combining observations from all three populations, green was the most frequent skin color, followed by orange, then yellow. However, skin color proportions differed between the populations.

Fruit flesh (mesocarp and/or endocarp) color also varied within each population (Table 1, Table S1). Combining observations from all three populations, the most frequent flesh color in the $F_{2}$ fruits was white, followed by white with some coloration in the endocarp. Occasionally there were fruits that had green or yellow flesh, and two fruits from the same plant from the PI 606066 X PI 163217 population had orange flesh. We found 17 fruits that had flesh with substantial coloration, and pooled these fruits (Table S1, Table 2) for RNA sequencing, along with a separate pool of 17 white fruits.

\section{Carotenoid pathway and DnaJ chaperone gene expression}

From the RNA sequences from the color and white pools, we were able to determine differential gene expression between the pools. 1889 genes had higher expression in the color pool, and 1292 genes had higher expression in the white pool (Supplementary Table S2). From among these DEGs, we focused on expression of genes in the carotenoid synthesis pathway (Table 3). Expression of some genes that are upstream of ß-carotene, such as PSY and carotene cis-trans isomerase (CRTISO) had slightly higher expression in the color pool, yet some other genes that are upstream of ß-carotene, such as zeta-carotene isomerase (ZISO) and zetacarotene desaturase (ZDS), had higher expression in the white pool. Likewise, expression of some genes downstream of ß-carotene were higher in the color pool, while others were lower in the white pool. Thus, there was no clear pattern to suggest that differences in carotenoid synthesis gene expression were involved in differences in fruit flesh color.

Since a mutation in the Or gene of cauliflower, which encodes a DnaJ chaperone protein, leads to carotenoid accumulation, we also checked for differential expression of DnaJ chaperone genes in the color and white pools. Nine DnaJ chaperone genes had higher 
expression in the color pool. However, none of these transcripts corresponded to the CsGy6G025020 gene that is most homologous to the Or gene.

\section{Enriched SNPs in each pool}

From the RNA sequencing reads, we were also able to identify SNP occurrences in the three color pool samples and the three white pool samples, relative to the reference genome (Table S3). Only one of the carotenoid synthesis transcripts had any SNPs; CsGy1G032330, which encodes ZDS, had three SNPS. However, these SNPS are unlikely to be involved in color accumulation, because although they were found mainly in the color pool, they were not homozygous. In addition to the carotenoid synthesis pathway, we checked for SNPs in DnaJ chaperone protein-encoding genes. Most interestingly, CsGy6G025020, which is homologous to the Or gene, had an indel SNP, an insertion of a $\mathrm{G}$, at position 923 . This indel was homozygous in all of the color pool samples. This insertion was not present in any of the white pool samples. In the deduced protein sequence from the reference and color pool transcripts, this insertion in the color pools would result in a translation frame shift followed by early termination of the protein (Fig. 3).

\section{Carotenoid analysis in orange and white fruits}

To determine whether the orange coloration of the cucumber flesh was due to $B$ carotene, we used HPLC to determine $ß$-carotene levels in an orange fruit, a green fruit, and a white fruit (Fig. 2B). The white fruit had only a trace of $ß$-carotene, while the orange fruit had much greater quantities, and the green fruit had an intermediate level (Fig. 4). In addition to the $\beta$-carotene peak, the green and orange fruits had several unidentified peaks that were eluted at earlier retention times. Thus, the colored fruits contained higher quantities of $\beta$ carotene than the white fruits, and also more of some unknown compounds.

\section{Discussion}

\section{Previous genetic basis for orange cucumber}

Our overall goal in this project was to identify genetic factors in cucumber that are responsible for orange fruit coloration. We confirmed that the orange coloration is due, at least in part, to ß-carotene (Fig. 4). In some instances, expression of PSY has been identified as a limiting factor in ß-carotene synthesis (Cazzonelli and Pogson, 2010). However, PSY expression and that of other genes in the carotenoid synthesis pathway do not seem to be likely causes of orange coloration, since there was no clear pattern of differential expression in either the color or white pools, upstream or downstream of ß-carotene (Table 3). In the closely related species Cucumis melo (melon), $\mathrm{F}_{3}$ families from a population with green-fleshed or orange-fleshed fruits were pooled by developmental time and fruit color (green or orange) (Chayut et al., 2015). 
Despite the differences in ß-carotene concentration, expression of carotenoid synthesis genes was similar between the green and orange pools, consistent with our results.

SNPs in carotenoid synthesis genes were not detected in this study, except for some in a $Z D S$ gene, which did not strictly segregate with the pools. In the previously studied orange cucumber fruits of the XIS lines, a mutation in $B C H 1$ was responsible for accumulation of $ß$ carotene in fruits (Qi et al., 2013). However, we did not detect any SNPs in this gene in the cucumber lines in this study. Therefore, it is unlikely that changes in expression or function of $B$ carotene synthesis or degradation genes underlie the orange coloration in fruits of these populations.

\section{The Orgene is mutated in the color pool}

In cauliflower, the BoOr mutation in a DnaJ chaperone protein led to formation of chromoplasts and accumulation of ß-carotene in florets (Lu et al., 2006). This cauliflower mutation resulted from a transposon. Similarly, in melon, a SNP in the Or gene resulted in an arginine to histidine substitution (Tzuri et al., 2015), which segregated perfectly with orange or green fleshed fruits (Chayut et al., 2015). In our study, we did detect an indel SNP in the homolog of the BoOr or CmOr genes, which was an extra $\mathrm{G}$ that resulted in a frame-shift and premature stop codon (Fig. 3). This mutation was homozygous all three samples in the orange pool. Thus, we believe it is likely to be necessary for orange coloration in these lines. The SNP in our cucumber samples occurred later in the translated protein than either the cauliflower or melon mutations. Thus, it seems that the Or gene may be quite sensitive to mutations, several of which at different positions (Lu et al., 2006, Tzuri et al., 2015) are enough to allow accumulation of $ß$-carotene inside chromoplasts that develop due to the mutation.

\section{Additional genetic factors}

Although our study indicates a genetic basis for orange cucumber that differs from that of the XIS cucumber lines, larger population sizes and/or advanced generations could be studied using the Or SNP as a genetic marker to calculate genetic ratios. Although the Or SNP was homozygous in the color pool, with larger populations, larger pools composed of only orange fruits could be made, and additional pools with green or yellow flesh could allow a finerscale dissection of gene expression and SNP differences that may be associated with flesh color. The PI 200815 line, which has yellow flesh and is one of the parents used in this study, was studied previously. One study attributed the yellow coloration to two recessive genes called $v$ and $w$ (Kooistra 1971), while a more recent study attributed the yellow coloration to a single recessive gene called yf (Lu et al., 2015). The current study design did not allow us to accurately quantify color and segregation ratios, but there were fruits with varying degrees of color (Table 1) and full orange fruits were rare (2 of 126), suggesting that polygenic inheritance is likely, and that the Or SNP is not the only factor. Although this study did not allow us to identify the $y f$ genes or other unknown genes, it seems clear that the $B C H 1$ gene is not the key factor in these populations, since there were no SNPs in it.

It might also be useful to study these populations separately, rather than pooling them. Because of small fruit numbers, our pools had uneven contributions of each parent in pools 
(Table 2). This pooling imbalance could have led us to miss other important DEGs or SNPs that might be more apparent in biparental populations. Based on our sampling of each population, there may be different important genes segregating in each one. For example, the PI $200815 \mathrm{X}$ PI 163217 population had predominantly white-fleshed fruits, while the PI 606066 X 163217 population had green+yellow flesh as the most frequent coloration, and the PI $606066 \mathrm{X} \mathrm{PI}$ 200815 had a large number of green-fleshed fruits (Table 1, Table S1). Thus, studying each population separately might yield additional information about the genetic basis of green, yellow, or orange flesh in cucumber fruits.

\section{Conclusions}

This study indicates that there are other sources of genetic variation for flesh color in cucumber, including the Or gene, and that it is not limited to the $\mathrm{BCH} 1$ gene that is responsible for orange flesh coloration in the XIS populations. This new information gives plant breeders additional germplasm to use for breeding ß-carotene-enhanced cucumbers. It should be possible to use multiple sources of $ß$-carotene accumulation (e.g. the $B C H 1$ mutant from XIS, Or mutant lines from these PI lines, and other unidentified genes) to develop new varieties with improved nutritional value and a novel appearance that might be popular with consumers. Additionally, our dataset could be useful for datamining for future studies.

\section{Acknowledgements}

This work was partially funded by a grant to BMW from the University of Nebraska Fleming Horticulture Research Fund. The authors thank Sam Polk for assistance with growing populations and sampling the fruits. The authors thank Edgar Cahoon for the use of the HPLC. The UNMC DNA Sequencing Core Facility receives partial support from the Nebraska Research Network In Functional Genomics NE-INBRE P20GM103427-14, The Molecular Biology of Neurosensory Systems CoBRE P30GM110768, The Fred \& Pamela Buffett Cancer Center P30CA036727, The Center for Root and Rhizobiome Innovation (CRRI) 36-5150-2085-20, and the Nebraska Research Initiative.

\section{References}

Bo K, Song H, Shen J, Qian C, Staub JE, Simon PW, Lou Q, Chen J. 2012. Inheritance and mapping of the ore gene controlling the quantity of beta-carotene in cucumber (Cucumis sativus L.) endocarp. Molecular Breeding 30, 335-344, 10.1007/s11032-011-9624-4.

Bolger AM, Lohse M, Usadel B. 2014. Trimmomatic: a flexible trimmer for Illumina sequence data. Bioinformatics 30, 2114-2120, 10.1093/bioinformatics/btu170.

Cazzonelli Cl, Pogson BJ. 2010. Source to sink: regulation of carotenoid biosynthesis in plants. Trends in Plant Science 15, 266-274, 10.1016/j.tplants.2010.02.003. 
Chayut N, Yuan H, Ohali S, Meir A, Yeselson Y, Portnoy V, Zheng Y, Fei Z, Lewinsohn E, Katzir N, Schaffer AA, Gepstein S, Burger J, Li L, Tadmor Y. 2015. A bulk segregant transcriptome analysis reveals metabolic and cellular processes associated with Orange allelic variation and fruit beta-carotene accumulation in melon fruit. BMC Plant Biology 15, 274, 10.1186/s12870015-0661-8.

Che G, Zhang X. 2019. Molecular basis of cucumber fruit domestication. Current Opinion in Plant Biology 47, 38-46, 10.1016/j.pbi.2018.08.006.

Cuevas HE, Song H, Staub JE, Simon PW. 2010. Inheritance of beta-carotene-associated flesh color in cucumber (Cucumis sativus L.) fruit. Euphytica 171, 301-311, 10.1007/s10681-0090017-2.

Diretto G, Welsch R, Tavazza R, Mourgues F, Pizzichini D, Beyer P, Giuliano G. 2007. Silencing of beta-carotene hydroxylase increases total carotenoid and beta-carotene levels in potato tubers. BMC Plant Biology 7, 11, 10.1186/1471-2229-7-11.

Kandlakunta B, Rajendran A, Thingnganing L. 2008. Carotene content of some common (cereals, pulses, vegetables, spices and condiments) and unconventional sources of plant origin. Food Chemistry 106, 85-89, 10.1016/j.foodchem.2007.05.071.

Kooistra E. 1971. Inheritance of fruit flesh and skin colors in powdery mildew resistant cucumbers (Cucumis sativus L). Euphytica 20, \&.

Langmead B, Salzberg SL. 2012. Fast gapped-read alignment with Bowtie 2. Nature Methods 9, U54, 10.1038/NMETH.1923.

Li H, Handsaker B, Wysoker A, Fennell T, Ruan J, Homer N, Marth G, Abecasis G, Durbin R, 1000 Genome Project Data Proc. 2009. The Sequence Alignment/Map format and SAMtools. Bioinformatics 25, 2078-2079, 10.1093/bioinformatics/btp352.

Li L, Yuan H. 2013. Chromoplast biogenesis and carotenoid accumulation. Archives of Biochemistry and Biophysics 539, 102-109, 10.1016/j.abb.2013.07.002.

Lu HW, Miao H, Tian GL, Wehner TC, Gu XF, Zhang SP. 2015. Molecular mapping and candidate gene analysis for yellow fruit flesh in cucumber. Molecular Breeding 35, 64, 10.1007/s11032015-0263-z.

Lu S, Van Eck J, Zhou X, Lopez AB, O'Halloran DM, Cosman KM, Conlin BJ, Paolillo DJ, Garvin DF, Vrebalov J, Kochian LV, Kupper H, Earle ED, Cao J, Li L. 2006. The cauliflower or gene encodes a DnaJ cysteine-rich domain-containing protein that mediates high levels of betacarotene accumulation. Plant Cell 18, 3594-3605, 10.1105/tpc.106.046417. 
Navazio JP, Simon PW. 2001. Diallel analysis of high carotenoid content in cucumbers. Journal of the American Society for Horticultural Science 126, 100-104.

Nisar N, Li L, Lu S, Khin NC, Pogson BJ. 2015. Carotenoid metabolism in plants. Molecular Plant 8, 68-82, 10.1016/j.molp.2014.12.007.

Qi J, Liu X, Shen D, Miao H, Xie B, Li X, Zeng P, Wang S, Shang Y, Gu X, Du Y, Li Y, Lin T, Yuan J, Yang X, Chen J, Chen H, Xiong X, Huang K, Fei Z, Mao L, Tian L, Staedler T, Renner SS, Kamoun S, Lucas WJ, Zhang Z, Huang S. 2013. A genomic variation map provides insights into the genetic basis of cucumber domestication and diversity. Nature Genetics 45, U149, 10.1038/ng.2801.

Robinson MD, McCarthy DJ, Smyth GK. 2010. edgeR: a Bioconductor package for differential expression analysis of digital gene expression data. Bioinformatics 26, 139-140, 10.1093/bioinformatics/btp616.

Simon PW, Navazio JP. 1997. Early Orange Mass 400, Early Orange Mass 402, and Late Orange Mass 404: High-carotene cucumber germplasm. HortScience 32, 144-145.

Tzuri G, Zhou X, Chayut N, Yuan H, Portnoy V, Meir A, Sa'ar U, Baumkoler F, Mazourek M, Lewinsohn E, Fei Z, Schaffer AA, Li L, Burger J, Katzir N, Tadmor Y. 2015. A 'golden' SNP in CmOr governs the fruit flesh color of melon (Cucumis melo). Plant Journal 82, 267-279, 10.1111/tpj.12814.

Waters BM, McInturf SA, Amundsen K. 2014. Transcriptomic and physiological characterization of the fefe mutant of melon (Cucumis melo) reveals new aspects of iron-copper crosstalk. New Phytologist 203, 1128-1145, 10.1111/nph.12911.

Zheng Y, Wu S, Bai Y, Sun H, Jiao C, Guo S, Zhao K, Blanca J, Zhang Z, Huang S, Xu Y, Weng Y, Mazourek M, Reddy UK, Ando K, McCreight JD, Schaffer AA, Burger J, Tadmor Y, Katzir N, Tang X, Liu Y, Giovannoni JJ, Ling K, Wechter WP, Levi A, Garcia-Mas J, Grumet R, Fei Z. 2019. Cucurbit Genomics Database (CuGenDB): a central portal for comparative and functional genomics of cucurbit crops. Nucleic Acids Research 47, D1136, 10.1093/nar/gky944. 
Tables

Table 1. Summary of skin and flesh colors in fruits of three cucumber $F_{2}$ populations.

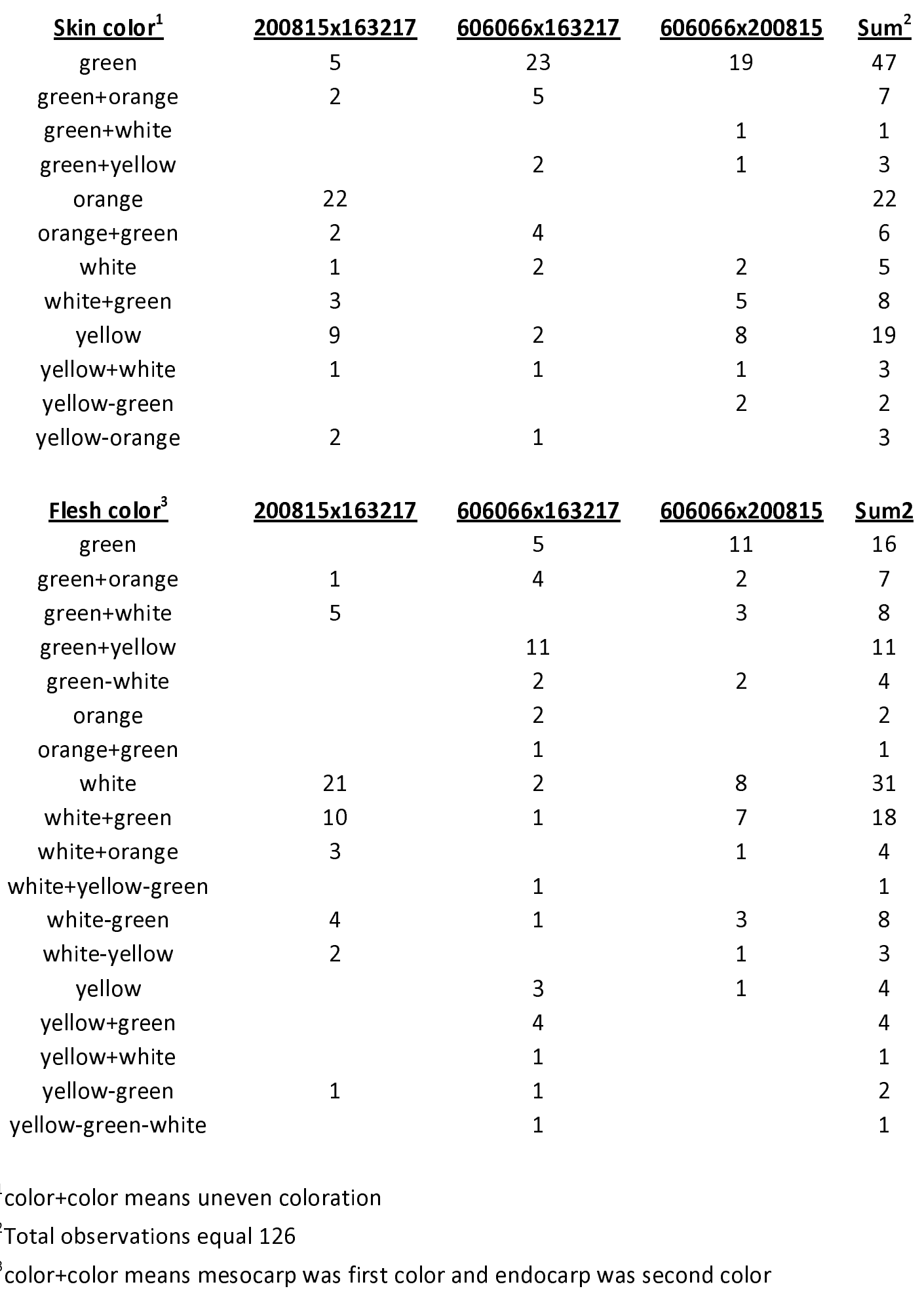


bioRxiv preprint doi: https://doi.org/10.1101/685289; this version posted June 27, 2019. The copyright holder for this preprint (which was not certified by peer review) is the author/funder, who has granted bioRxiv a license to display the preprint in perpetuity. It is made available under aCC-BY-NC-ND 4.0 International license.

Table 2. Contribution of each parent to color and white flesh pools.

\section{Color pool}

Parent No. lines \% of total

$200815 \quad 8 \quad 23.5$

$\begin{array}{lll}606066 & 14 & 41.2\end{array}$

$163217 \quad 12 \quad 35.3$

White pool

$\begin{array}{cccc}\underline{\text { Parent }} & & \text { No. lines } & \text { \% of tota } \\ 200815 & 15 & 44.1 \\ 606066 & 9 & 26.5 \\ 163217 & 10 & 29.4\end{array}$


Table 3. Differentially expressed genes of the carotenoid synthesis pathway.

\section{GeneID Description}

CsGy4G007210.1 Phytoene synthase 3 (PSY)

CsGy4G003270.1 15-cis-zeta-carotene isomerase (ZISO)

CsGy1G032330.1 Zeta-carotene desaturase (ZDS)

CsGy3G006450.1 prolycopene isomerase 1 (CRTISO)

CsGy4G000680.1 Lycopene beta cyclase (b-LCY)

CsGy3G007100.1 Cytochrome P450, putative (BCH)

CsGy3G017310.1 Beta-carotene hydroxylase (BCH) (Ore gene)

CsGy2G014000.1 Zeaxanthin epoxidase (ZEP)

CsGy2G008070.1 violaxanthin de-epoxidase (VDE)

CsGy2G015170.1 violaxanthin de-epoxidase (VDE)

CsGy4G006910.1 Carotenoid cleavage dioxygenase 4 (CCD)

CsGy4G007460.1 9-cis-epoxycarotenoid dioxygenase (NCED)

CsGy6G035690.1 9-cis-epoxycarotenoid dioxygenase (NCED)

\begin{tabular}{|c|c|}
\hline$\underline{\log F C^{1}}$ & Position $^{2}$ \\
\hline 0.66 & upstream \\
\hline-0.79 & upstream \\
\hline-0.61 & upstream \\
\hline 0.74 & upstream \\
\hline-0.86 & upstream \\
\hline 1.03 & downstream \\
\hline-1.28 & downstream \\
\hline-0.99 & downstream \\
\hline 0.74 & downstream \\
\hline 1.49 & downstream \\
\hline-2.03 & downstream \\
\hline-1.29 & downstream \\
\hline-2.88 & downstream \\
\hline
\end{tabular}

${ }^{1}$ Positive logFC means higher in color pool, negative logFC means higher in white pool

${ }^{2}$ Position in carotenoid synthesis pathway relative to beta-carotene 


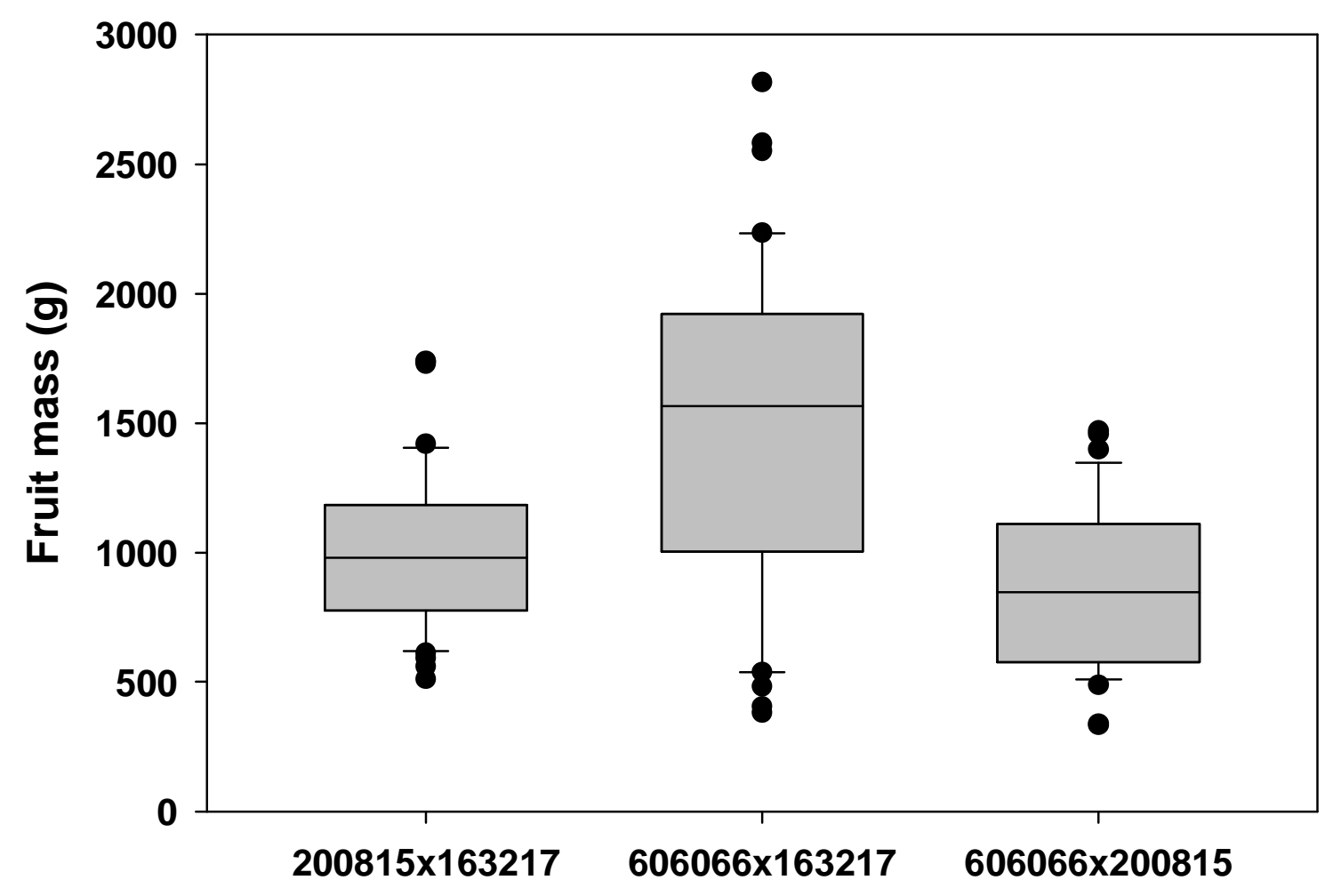

Figure 1. Cucumber fruit size (mass) of fruits from each of three $F_{2}$ populations used in this study. Two fruits were collected from each plant. Numbers indicate each PI line parent. 

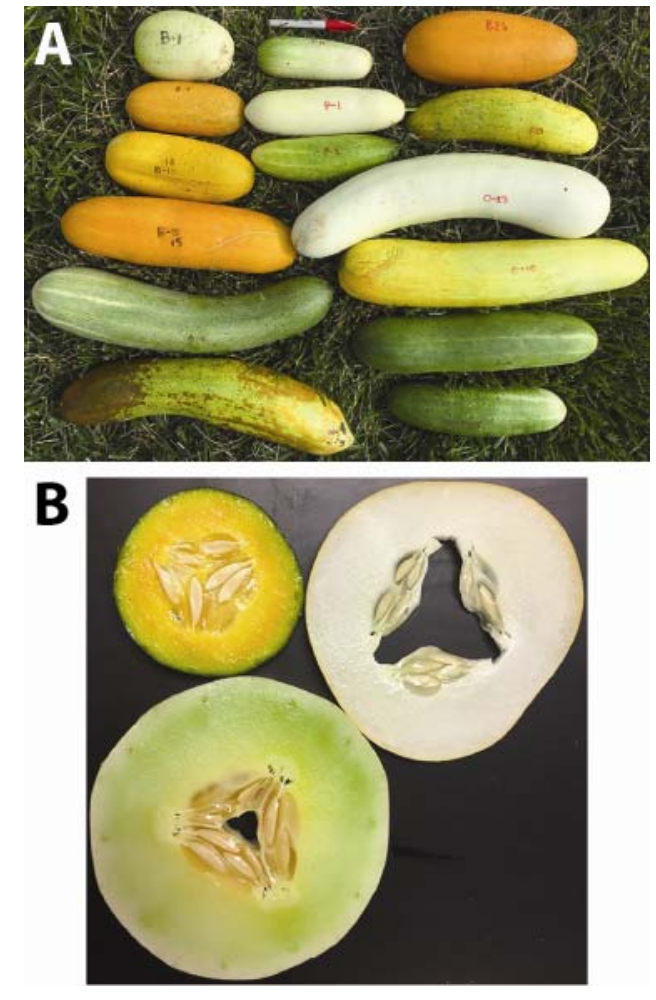

Figure 2. Diversity of cucumber fruit size, shape, and color. A, Photograph of several fruits from each $F_{2}$ population. B, Cross-section photograph of orange, green, and white fruits used for HPLC analysis of beta-carotene. 
CsGy6G025020.1_Ref CsGy6G025020.1_Color

B.oleracea or MELO3C0054 49

CsGy6G025020.1_Ref CsGy6G025020.1_Color B.oleracea_Or

MELO3C0054 49

CsGy6G025020.1_Ref CsGy6G025020.1_Color B.oleracea_Or MELO3C0054 49

CsGy6G025020.1_Ref CsGy6G025020.1_Color B.oleracea_Or MELO3C005449

CsGy6G025020.1_Ref CsGy6G025020.1_Color B.oleracea_Or MELO3C0054 $\overline{4} 9$

CsGy6G025020.1 Ref CsGy6G025020.1 Color B. oleracea_Or MELO3C0054 49
MSLPSIFAPSSHFPLPISHFKPSRHCRASIFLSIDYRC--C-KSIYASS-- - - RSISIR 52 MSLPS IFAPSSHFPLPISHFKPSRHCRASIFLSIDYRC--C-KSIYASS-- - - RSISIR 52 MS - - CLGR ILSVSYP PDPYGSR - - - - - - - - - - - - LSVSKLSS - - PGRNRRLRWR 38 - - - - - MDRVLVASYP INHLIRPH - - - - -SFRIDYCWSTCFTSRLNSGKERQKLSSRWR 48

SPRICAS- - - - SSDG-ASASVPSDSDNTSSNFCI IEGPETVQDFVQMQFQEIQDNIRSR 106 SPRICAS-- ---SSDG-ASASVPSDSDNTSSNFCIIEGPETVQDFVQMQFQEIQDNIRSR 106 FTALDSDS - - - - - - -SSLDSDSSDKFAAGFCI IEGPETVQDFAKMQLQEIQDNIRSR 88 WRSMASDSTDSSSSSSFAPSVESDPSDKTSASFCI IEGPETVQDFAKMELQEIQENIRSR 108

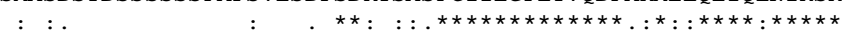

RNKIFLLMEEVRRLRIQQRLKNLKPIDENDIEEANEMPDIPSSIPFLPHVTPKTLKQQYL 166 RNKI FLLMEEVRRLRIQQRLKNLKPIDENDI EEANEMPDI PSS I F LPHVTPKTLKQQYL 166 RNKIFLHMEEVRRLRIQQRIRNTE-LGIIDEEQEHELPNFPSFIPFLPPLTAANLRVYYA 147 RNKIFLHMEEVRRLRIQQRI KNAE-LGISKEERENELPNFPSFIPFLPPLSSENLKLYYV 167

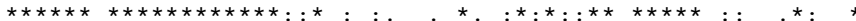

TSLSVIWGI IVFGGLIAPTLELKLGLGGTSYEDF IRNMHLPMQLSQVDPIVASFSGGAVG 226 TSLSVIWGI IVFGGLIAPTLELKLGLGGTSYEDF IRNMHLPMQLSQVDPIVASFSGGAVG 226 TCFSLIAGI ILFGGLLAPTLELKLGIGGTSYKDF IQSLHLPMQLSQVDP IVASFSGGAVG 207 TCYSLIAGI ILFGGLLAPTLELKLGLGGTSYEDF IRSVHLPMQLSQVDPIVASFSGGAVG 227

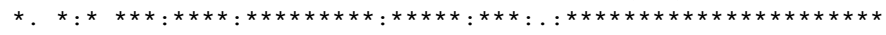

VISALMLIEANNVEQQEKKRCKYCHGTGYLACARCSSSGVCLSADPISLSASSSRPLRMP 286 VISALMLIEANNVEQQEKKRCKYCHGTGYLACARCSSSGVCLSADPISLSASSSRPLRMP 286 VISALMVVEVNNVKQQEHKRCKYCLGTGYLACARCSSTGSLIISEPVSAIAGGNHSVSTS 267 VISALMVVEVNNVKQQEHKRCKYCLGTGYLACARCSNTGALVLIEPVSTLNGEHQPLSLP 287

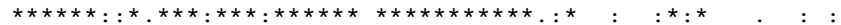

KTQRCLNCSGAGKVMCPTCLCTGMLMASEHDPRFDPFD 324 KTQRCLNCSGAGKVMCPTCLCTGDVDGK- - - - - - - 314 KTERCSNCSGAGKVMCPTCLCTGMAMASEHDPRIDPFL 305 KTERCQNCSGSGKVMCPTCLCTGMAMASEHDPRIDPFD 325

Figure 3. ClustalW alignment of deduced amino acid sequences of Orange (Or) genes from cucumber, cauliflower, and melon. CsGy6G025020.1_Ref, deduced sequence from reference transcriptome for Or homolog; CsGy6G025020.1_Color, deduced sequence from color pool with $\mathrm{G}$ indel at base 923 . Frameshift occurs at AA 310; B.oleracea_Or, deduced sequence from wild-type Or gene of cauliflower; MELO3C005449, deduced sequence from melon Or gene. 

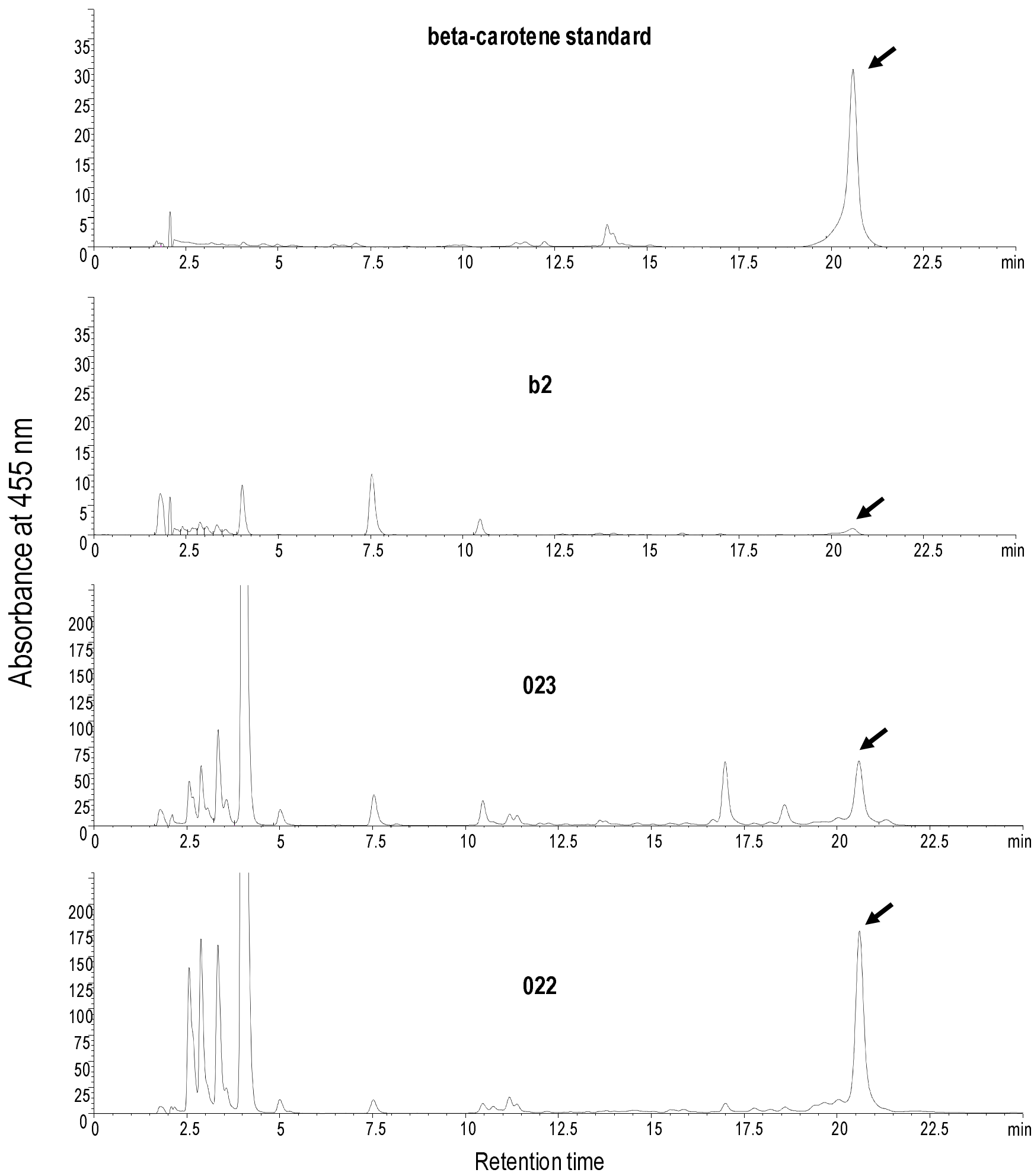

Figure 4. HPLC output for carotenoid analysis. Upper trace, beta-carotene standard; b2 trace, white-fleshed cucumber sample; 023 trace, green-fleshed cucumber sample; 022 trace, orange-fleshed cucumber sample. See Fig. 2B for photograph of white, green, and orange cucumbers. 